\title{
BRATTELI-VERSHIK MODELS FOR CANTOR MINIMAL SYSTEMS ASSOCIATED TO INTERVAL EXCHANGE TRANSFORMATIONS
}

\author{
RICHARD GJERDE and ØRJAN JOHANSEN
}

\begin{abstract}
We construct Bratteli-Vershik models for minimal interval exchange transformations. We use this to show that the interval exchange transformations over quadratic fields, recently studied by Boshernitzan and Carroll, actually are (conjugate to) substitution minimal systems. We also prove a partial converse to this. Furthermore, these systems are orbit equivalent to Sturmian systems.
\end{abstract}

\section{Minimal interval exchange transformations and their associated Cantor minimal systems}

Let $\xi=\left(\Delta_{1}, \ldots, \Delta_{k}\right)$ be a partition of $[0,1)$ into $k \geq 2$ disjoint semi-intervals (of the form $[a, b)$ ) numbered from left to right, and let $\pi=\left(\pi_{1}, \ldots, \pi_{k}\right.$ ) be a permutation of the numbers $(1,2, \ldots, k)$. A transformation $T:[0,1) \rightarrow[0,1)$, which is a translation $T_{\alpha_{i}}(x)=x+\alpha_{i} \quad(\bmod 1)$ on each of the semi-intervals $\Delta_{i}$ (the number $\alpha_{i}$ depends on $i$ ), such that

$$
\left\{T \Delta_{i}=\Delta_{i}^{\prime}: i \in\{1, \ldots, k\}\right\}
$$

is a semi-interval partition of $[0,1)$, where the $\Delta_{i}^{\prime}$ adhere to each other in the order $\Delta_{\pi_{1}}^{\prime}, \ldots, \Delta_{\pi_{k}}^{\prime}$, is called an interval exchange transformation on $k$ intervals.

The notion of an interval exchange transformation was introduced by Keane [8]. For a more complete discussion of interval exchange transformations we refer to [2, Chapter 5].

We will assume throughout that the transformation $T$ is minimal in the sense that the orbit under $T$ of any point in $[0,1)$ is dense in $[0,1)$. An obvious necessary condition for $T$ to be minimal is that $\pi$ be irreducible, i.e. $\pi$ does not map any segment $\{1,2, \ldots, j\}, j<k$, into itself. A sufficient condition is the so-called infinite distinct orbit condition; i.e. the orbit of any of the left end points of the intervals is infinite, and for points other than 0 , the orbits do not intersect [2, Chapter 5, $\$ 4$, Theorem 1]. 
Let $T:[0,1) \rightarrow[0,1)$ be a minimal interval exchange transformation on $k$ intervals. We want to include $[0,1)$ densely in a Cantor set $X$ and find a minimal homeomorphism $\phi: X \rightarrow X$ such that $\left.\phi\right|_{[0,1)}=T$. Let $\xi=\left\{\Delta_{1}, \ldots, \Delta_{k}\right\}$ be the intervals associated to $T$. Let $\mathscr{L}(T)=\left\{d_{1}, d_{2}, \ldots, d_{k}\right\}$ be the set of left endpoints of the intervals. It is clear that $T$ is continuous from the right, but discontinuous from the left at $d_{i} \neq 0$. We remedy this in the following fashion. Let $\mathscr{D}(T)$ denote the $T$-orbits of all $d_{i}$, and let

$$
X=([0,1) \backslash \mathscr{D}(T)) \bigcup\left\{x^{+}, x^{-}: x \in \mathscr{D}(T)\right\}
$$

where $0^{-}=1$ and $0^{+}=0$. Letting $x^{-}<x^{+}$for each $x \in \mathscr{D}(T)$ (with the exception that $0^{-} \geq x$ for all $x$ ), there is an obvious extension of the linear ordering on $[0,1)$ to a linear ordering on $X$. We endow $X$ with the order topology and get a Cantor set, since $\mathscr{D}(T)$ is dense in $[0,1)$ by minimality of $T$. We may include $[0,1)$ in $X$ by mapping $x$ in $\mathscr{D}(T)$ to $x^{+}$. We define $\phi: X \rightarrow X$ by $\phi(y)=T(y)$ if $y \in[0,1) \backslash \mathscr{D}(T)$ and if $x \in \mathscr{D}(T), \phi\left(x^{-}\right)=T(x)^{-}$and $\phi\left(x^{+}\right)=T(x)^{+}$. The interval $\Delta_{i}=\left[a_{i}, b_{i}\right)$ in $[0,1)$ corresponds to the clopen "interval" $\left[a_{i}^{+}, b_{i}^{-}\right]$in $X$.

We will henceforth call $(X, \phi)$ an interval exchange transformation on $k$ intervals (associated to $T$ ).

\section{Bratteli diagrams and dimension groups, ordered Bratteli diagrams and Bratteli-Vershik models.}

We give a brief survey of Bratteli diagrams and how they are employed to give models for Cantor minimal systems. We also state some basic facts about dimension groups and their relation to Bratteli diagrams and to Cantor minimal systems. For further details, the reader should consult the references [3], [4], [6], [7].

\subsection{Bratteli diagrams}

Definition 2.1. A Bratteli diagram is an infinite directed graph $(V, E)$, where $V$ is the vertex set and $E$ is the edge set. These sets are partitioned into non-empty disjoint finite sets $V=V_{0} \cup V_{1} \cup \ldots$ and $E=E_{1} \cup E_{2} \cup \ldots$, where $V_{0}=\left\{v_{0}\right\}$ is a one-point set. There are two maps $r, s: E \rightarrow V$ such that $r\left(E_{n}\right) \subseteq V_{n}$ and $s\left(E_{n}\right) \subseteq V_{n-1}$ for $n \in \mathrm{N}$. Furthermore, $s^{-1}(v) \neq \varnothing$ for all $v \in V$ and $r^{-1}(v) \neq \varnothing$ for all $v \in V \backslash V_{0}$. We call $r$ and $s$ the range map and the source map of $(V, E)$, respectively. We say that $u \in V_{n}$ is connected to $v \in V_{n+1}$ if there is an edge $e \in E_{n}$ such that $s(e)=u$ and $r(e)=v$.

If $V_{n}=\left\{u_{1}, \ldots, u_{k}\right\}$ and $V_{n+1}=\left\{v_{1}, \ldots, v_{m}\right\}$, we define an $m \times k$ matrix $A_{n}=\left(a_{i j}\right)$, where $a_{i j}$ is the number of edges connecting $v_{i}$ to $u_{j}$. We call $A_{n}$ the $n$th incidence matrix of $(V, E)$. 
Given $e_{k} \in E_{k}, e_{k+1} \in E_{k+1}, \ldots, e_{k+m} \in E_{k+m}$ such that $r\left(e_{i}\right)=s\left(e_{i+1}\right)$ for $i=k, k+1, \ldots, k+m-1$, we call the sequence $\left(e_{k}, \ldots, e_{k+m}\right)$ a path (of length $m+1)$ in $(V, E)$ starting at $s\left(e_{k}\right) \in V_{k-1}$ and terminating at $r\left(e_{k+m}\right) \in V_{k+m}$. In the same manner, we define infinite paths $\left(e_{1}, e_{2}, e_{3}, \ldots\right)$, starting from the top vertex $v_{0} \in V_{0}$.

Let $\left\{n_{k}\right\}_{k=0}^{\infty}$ be a subsequence of $\{0,1,2, \ldots\}$, where we assume that $n_{0}=0$. We telescope $(V, E)$ into a new Bratteli diagram $\left(V^{\prime}, E^{\prime}\right)$ by letting $V_{k}^{\prime}=V_{n_{k}}$ and letting $A_{k}^{\prime}=A_{n_{k+1}-1} A_{n_{k+1}-2} \cdots A_{n_{k}}$ be the new incidence matrices. Notice that $E^{\prime}, r^{\prime}$, and $s^{\prime}$ are obtained in a natural way from the incidence matrices $A_{k}^{\prime}$. Furthermore, we see that the edges $E_{k}^{\prime}$ from $V_{k-1}^{\prime}$ to $V_{k}^{\prime}$ correspond to the paths from $V_{n_{k-1}}$ to $V_{n_{k}}$ in $(V, E)$.

A Bratteli diagram is simple if it can be telescoped so that all the incidence matrices have only (strictly) positive entries.

\subsection{Ordered Bratteli diagrams}

For each $v \in V \backslash V_{0}$ we may give $r^{-1}(v)$ a linear ordering. This induces a partial order $\geq$ in $E$, and we obtain an ordered Bratteli diagram, which we denote by $(V, E, \geq)$. Let $E_{\max }$ and $E_{\min }$ denote the set of maximal and minimal edges, respectively. An infinite path is maximal (minimal) if all the edges making up the path are elements of $E_{\max }\left(E_{\min }\right)$. An ordered Bratteli diagram is properly ordered if it is simple and if it has a unique maximal and a unique minimal path, denoted respectively by $x_{\max }$ and $x_{\min }$. The partial order induces a (lexicographic) order on infinite cofinal paths. Specifically, if $x=\left(e_{1}, e_{2}, \ldots\right) \neq x_{\max }$, let $k$ be the smallest integer such that $e_{k} \notin E_{\max }$. Let $f_{k}$ be the successor of $e_{k}$ (and so $r\left(e_{k}\right)=r\left(f_{k}\right)$ ). Then the successor of $x$ is the path $\left(f_{1}, f_{2}, \ldots, f_{k}, e_{k+1}, e_{k+2}, \ldots\right)$, where $f_{1}, \ldots, f_{k-1} \in E_{\min }$.

An ordered Bratteli diagram is said to be left/right ordered (or vertex ordered) if whenever $e_{1} \geq e_{2}$ (hence necessarily $\left.r\left(e_{1}\right)=r\left(e_{2}\right)\right)$ and $s\left(e_{1}\right)=v_{i}$, $s\left(e_{2}\right)=v_{j}$, we have $i \geq j$. A left/right ordering is not preserved by telescoping, as is easily seen by examples.

\subsection{Bratteli-Vershik models for Cantor minimal systems}

Let $X$ be the set of all (infinite) paths associated to a properly ordered Bratteli diagram $(V, E, \geq)$. A cylinder set consists of those (infinite) paths that agree from the top vertex $v_{0}$ to some $v \in V_{n}, n \in \mathrm{N}$. It is easily seen that if we topologize $X$ by choosing as a basis of open sets the cylinder sets, then $X$ becomes a Cantor set, and the cylinder sets will be clopen sets. (We will tacitly assume that the Bratteli diagrams we consider are non-trivial, avoiding the cases where $X$ is a finite set.)

Let $\phi: X \rightarrow X$ be the map that takes an infinite path to its successor in the lexicographic ordering. This is well-defined for all paths except for the 
unique maximal path $x_{\max }$, and we define $\phi\left(x_{\max }\right)=x_{\min }$, the unique minimal path. It is straightforward to verify that $\phi$, called the Vershik map associated to $(V, E, \geq)$, is a minimal homeomorphism. Hence $(X, \phi)$ is a Cantor minimal system which we call the Bratteli-Vershik system associated to $(V, E, \geq)$.

It is a theorem [7, Theorem 4.7] that any Cantor minimal system $(Y, \psi)$ is conjugate to a Bratteli-Vershik system, which we will call a Bratteli-Vershik model for $(Y, \psi)$. We briefly recapitulate the construction. We start by choosing a decreasing sequence $\left\{C_{n}\right\}_{n=0}^{\infty}$ of clopen sets, where $C_{0}=Y$, shrinking down to a base point $y$, and constructing towers over these determined by first returns. The floors of the towers over $C_{n}$ will be a finite partition $\mathscr{P}_{n}$ of $Y$ consisting of clopen sets, which we will call the Kakutani-Rohlin partition associated to $C_{n}$. The clopen set $C_{n}$ will correspond to level $n$ in the properly ordered Bratteli diagram $(V, E, \geq)$ we construct. Each tower over $C_{n}$ corresponds to a vertex in $V_{n}$, and the way towers over $C_{n+1}$ traverse towers over $C_{n}$ determines the edges $E_{n+1}$ and the orders on these. If a tower over $C_{n}$ corresponds to the vertex $v \in V_{n}$, then the height of the tower equals the number of paths from the top vertex $v_{0} \in V_{0}$ to $v$. In the general case, we may have to subdivide vertically the various towers to make sure that the KakutaniRohlin partition $\mathscr{P}_{n+1}$ is a refinement of $\mathscr{P}_{n}$ and that the floors of the towers generate the topology of the space. In our situation, such a subdivision will automatically be achieved by the way we construct the towers. By the above construction, the base point $y$ will correspond to the unique minimal path $x_{\min }$ of the resulting Bratteli-Vershik system. (Note: In the references [6], [7], the roles of the base and top floors are reversed, so that there $y$ will correspond to $x_{\max }$.) It is important that the Bratteli-Vershik system we get is the same up to conjugacy regardless of the choice of base point and the choice of the sets shrinking down to the base point. This fact yields a great deal of freedom when we are going to do the construction for concrete examples. A judicious choice of the shrinking sequence may hugely simplify the construction and yield a "nice" Bratteli-Vershik model.

\subsection{Dimension groups}

To the Bratteli diagram $(V, E)$ is associated a dimension group $G=K_{0}(V, E)$. In fact, to $(V, E)$ is associated the system of (simplicially) ordered groups (cf. Definition 2.1)

$$
Z^{\left|V_{0}\right|} \stackrel{A_{0}}{\longrightarrow} Z^{\left|V_{1}\right|} \stackrel{A_{1}}{\longrightarrow} Z^{\left|V_{2}\right|} \stackrel{A_{2}}{\longrightarrow} \cdots,
$$

where the order-preserving homomorphisms are given by matrix multiplication with the incidence matrices $\left\{A_{i}\right\}_{i=0}^{\infty}$. ( $Z^{\left|V_{i}\right|}$ denotes the $\left|V_{i}\right| \times 1$ column vectors over Z.) By definition $K_{0}(V, E)$ is the direct limit of the system endowed with the induced order. The ordered group $K_{0}(V, E)$ has a distinguished order unit, 
namely the element of the positive cone $G^{+}=K_{0}(V, E)^{+}$corresponding to the element $1 \in Z^{\left|V_{0}\right|}=Z$.

Effros, Handelman, and Chen have given an abstract characterization of dimension groups [4].

It has been proved [7] that the family of simple dimension groups coincides with the family

$$
\left\{\left(K^{0}(Y, \psi), K^{0}(Y, \psi)^{+}\right):(Y, \psi) \text { a Cantor minimal system }\right\},
$$

where $K^{0}(Y, \psi)=C(Y, \mathrm{Z}) / \partial_{\psi} C(Y, \mathrm{Z})$, with the induced order from $C(Y, \mathrm{Z})$, and where $C(Y, \mathrm{Z})$ denotes the continuous functions on $Y$ with values in $\mathrm{Z}$. Here

$$
\partial_{\psi}: C(Y, \mathrm{Z}) \rightarrow C(Y, \mathrm{Z})
$$

is the coboundary map defined by $\partial_{\psi}(f)=f-f \circ \psi^{-1}$. (There is a distinguished order unit 1 for $K^{0}(Y, \psi)$, corresponding to the constant function 1.) In fact, $K^{0}(Y, \psi)$ is order isomorphic to the dimension group $K_{0}(V, E)$, with 1 mapping to the distinguished order unit of $K_{0}(V, E)$, where $(V, E, \geq)$ is the properly ordered Bratteli diagram associated to $(Y, \psi)$. In [6] it is shown that $K^{0}(Y, \psi)$ is intimately related to the orbit structure of $(Y, \psi)$.

\section{The Bratteli-Vershik model for interval exchange systems}

In this section we show the following result.

THEOREM 3.1. Let $(X, \phi)$ be a minimal interval exchange transformation on $k$ intervals associated to $T$. Then the associated Cantor minimal system corresponds to a simple ordered Bratteli diagram $(V, E, \geq)$, where

(i) $\left|V_{1}\right|=k$ and $\left|V_{i}\right|-\left|V_{i+1}\right| \in\{0,1\}$ for all $i \geq 1$.

(ii) $\lim _{i \rightarrow \infty}\left|V_{i}\right|-1$ exists and is equal to the number of distinct orbits in $\mathscr{D}(T)$.

(iii) Any incidence matrix $A_{i}$ between levels $i$ and $i+1$ for $i \geq 1$ and $\left|V_{i}\right|=\left|V_{i+1}\right|$, has determinant 1 . More precisely, it is of the form

$$
A_{i}=\left(\begin{array}{cccccccc}
1 & 0 & \cdots & 0 & 0 & \cdots & 0 & s_{1} \\
0 & 1 & \cdots & 0 & 0 & \cdots & 0 & s_{2} \\
\vdots & \vdots & & \vdots & \vdots & & \vdots & \vdots \\
0 & 0 & \cdots & 1 & 0 & \cdots & 0 & s_{l} \\
0 & 0 & \cdots & 1 & 0 & \cdots & 0 & s_{l+1} \\
0 & 0 & \cdots & 0 & 1 & \cdots & 0 & s_{l+2} \\
\vdots & \vdots & & \vdots & \vdots & & \vdots & \vdots \\
0 & 0 & \cdots & 0 & 0 & \cdots & 1 & s_{k}
\end{array}\right),
$$


where $s_{i} \in\{0, m, m+1\}, s_{l}=m$ and $s_{l+1}=m+1$ for some $m \in Z_{+}$. When $\left|V_{i}\right|-\left|V_{i+1}\right|=1$, row $l+1$ is omitted. All entries of $A_{0}$ are 1 (i.e. there is a single edge from the top vertex to each of the vertices at level 1).

(iv) The diagram is endowed with the left/right ordering.

Proof. We choose 0 as our base point. We must describe clopen sets $\left\{A_{n}\right\}_{n=1}^{\infty}$ so that $A_{1} \supset A_{2} \supset A_{3} \supset \cdots$ and $\bigcap_{n=1}^{\infty} A_{n}=\{0\}$. Let $A_{1}=\bigcup_{i=1}^{k-1} \Delta_{i}$. It is quite easy to show that the map $\phi_{1}$ on $A_{1}$ induced by $\phi$ is also an interval exchange transformation on $k_{1} \in\{k-1, k\}$ intervals [2, Ch. 5, $\S 3$, Lemma 2]. In other words, we have a semi-interval partition $\left\{\Delta_{1}^{1}, \Delta_{2}^{1}, \ldots, \Delta_{k_{1}}^{1}\right\}$ of $A_{1}$ so that $\phi_{1}$ is an interval exchange transformation on these intervals. Now we let $A_{2}=\bigcup_{i=1}^{k_{1}-1} \Delta_{i}^{1}$. The induced map $\phi_{2}$ is similarly an interval exchange transformation on $k_{2} \in\left\{k_{1}-1, k_{1}\right\}$ intervals $\left\{\Delta_{1}^{2}, \Delta_{2}^{2}, \ldots, \Delta_{k_{2}}^{2}\right\}$. We carry on by induction. The $k_{n+1}$ intervals of $A_{n+1}$ are formed by the $k_{n}-1$ first intervals of $A_{n}$ with the additional condition that one of these intervals may be split into two as shown in Figure 1.
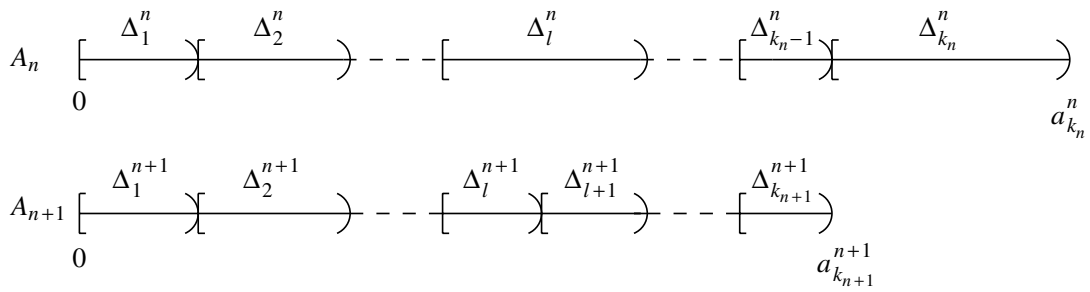

Figure 1. The intervals of $A_{n}$ and $A_{n+1}$

We will show in more detail below how this comes about.

REMARK. The only way $k_{n+1}<k_{n}$ can occur is when no interval is split during the passage to the induced system. This happens exactly when the left end point of some interval is mapped by the induced mapping onto the same point as the left end point of the removed, rightmost interval. This means that these end points are in the same orbit. Conversely, as the intervals shrink to $\{0\}$, whenever two left end points are in the same orbit the construction eventually has to map them to the same point (and thus remove one of the corresponding intervals), except that 0 and $\phi^{-1}(0)$ are kept separate. This shows point (ii) of the theorem.

Obviously, $k_{n}$ is eventually constant, and so by passing to an induced system we may assume without loss of generality that $k_{n}=k$ for all $n$, and we will do so hereafter. 
LemMA 3.2. Let $\left\{A_{n}\right\}_{n=1}^{\infty}$ be a decreasing sequence of subintervals constructed from a minimal interval exchange transformation on $k$ intervals as described above. Then $\bigcap_{n=1}^{\infty} A_{n}=\{0\}$.

Proof. Assume that $\bigcap_{n=1}^{\infty} A_{n} \neq\{0\}$. It follows easily that $\bigcap_{n=1}^{\infty} A_{n}=$ $[0, a]$ for some $0<a<1$, which cannot be isolated from the right. Let $m$ be the least natural number so that $\phi^{-m}(a) \in[0, a)$. By the continuity of $\phi$, choose $b>a$ such that $\phi^{-m}([a, b)) \subset[0, a)$ and $\phi^{-i}([a, b)) \subset(b, 1]$ for all $i \in\{1,2, \ldots, m-1\}$. Since $\bigcap_{n=1}^{\infty} A_{n}=[0, a]$, it is clear that there exists an $N \in \mathrm{N}$ such that $\Delta_{k}^{l} \subset[a, b)$ for each $l \geq N$.

Consider $x \in[a, b)$. Then iterations of $\phi$ return $x$ to $[0, a)$ before $[a, b)$, and so $x$ cannot be a splitting point of any interval $\Delta_{j}^{l}, l \geq N, j \in\{1,2, \ldots, k\}$. Thus the set $\left\{\Delta_{j}^{l} \mid \Delta_{j}^{l} \cap[a, b) \neq \emptyset, j=1, \ldots, k\right\}$ must decrease by 1 in cardinality for each level $l \geq N$, eventually reaching 0 and so $\Delta_{k}^{l} \not \subset[a, b)$, a contradiction.

We also need to assure that the tower floors generate the topology. It is sufficient to show that any pair of points are separated at some level. By Lemma 3.2, 0 is the only point which is at the base floor of all its towers, and it follows immediately that $\phi^{-1}(0)$ is the only point which is at the top floor of all its towers. Thus these points are separated from all others. We shall need the following:

Lemma 3.3. For $x \neq y \in X$ there exists $n \in Z$ such that $\phi^{n}(x)$ and $\phi^{n}(y)$ are in different intervals.

Proof. If $x$ and $y$ are equal to $z^{-}$and $z^{+}$for some $z \in[0,1)$, choose $n$ so that $T^{n}(z)$ is one of the original interval endpoints. Otherwise, choose $N \in \mathrm{N}$ by minimality such that $\phi^{N}(y)<|y-x|$. Since $\phi$ preserves distance and order within each interval, $\phi^{n}(x)$ and $\phi^{n}(y)$ must be in different intervals for some $0 \leq n<N$.

Assume ad absurdum that $x \neq y$ are not separated by the tower floors at any level, and let $n$ be as in Lemma 3.3. There are 3 possibilities:

1. From some level on, $x$ and $y$ are in the $k^{\prime}$ th floor from the bottom, $k<|n|$. Then it follows that $\phi^{-k}(x)$ and $\phi^{-k}(y)$ are both at the base floor of all towers, and are equal to 0 .

2. From some level on, $x$ and $y$ are in the $k^{\prime}$ th floor from the top, $k<|n|$. Similarly, they are then both equal to $\phi^{-k-1}(0)$.

3. There is some level such that $x$ and $y$ are at least $|n|$ floors from the top and bottom floors. Since they are in the same element of the tower partition, $\phi^{n}(x)$ and $\phi^{n}(y)$ must also be in the same element, but this contradicts their being in different intervals. 
Having shown that the $A_{n}$ fulfill our requirements, we use them to construct an ordered Bratteli diagram which models our Cantor system. At each level we get $k$ vertices corresponding to the $k$ intervals of the induced interval exchange transformation from $\phi$ on each $A_{n}$. This proves the first point of the Theorem.

We have two different possibilities for the permutation of the $k$ intervals in $A_{n}$ as shown in Figure 2.
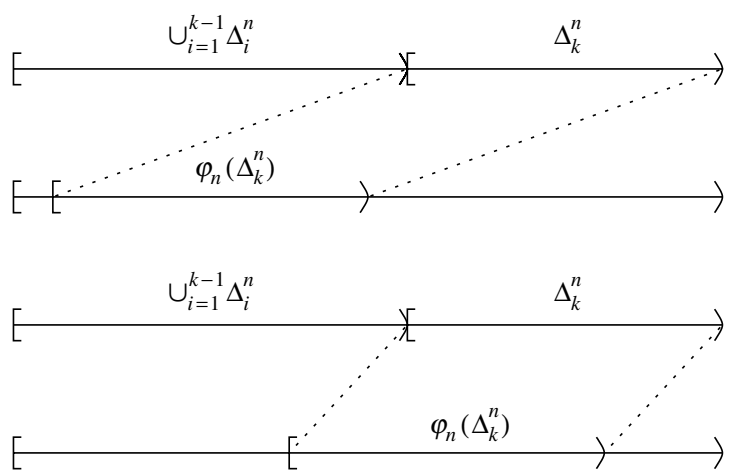

Figure 2. The two ways $\Delta_{k}^{n}$ may be moved by $\phi_{n}$.

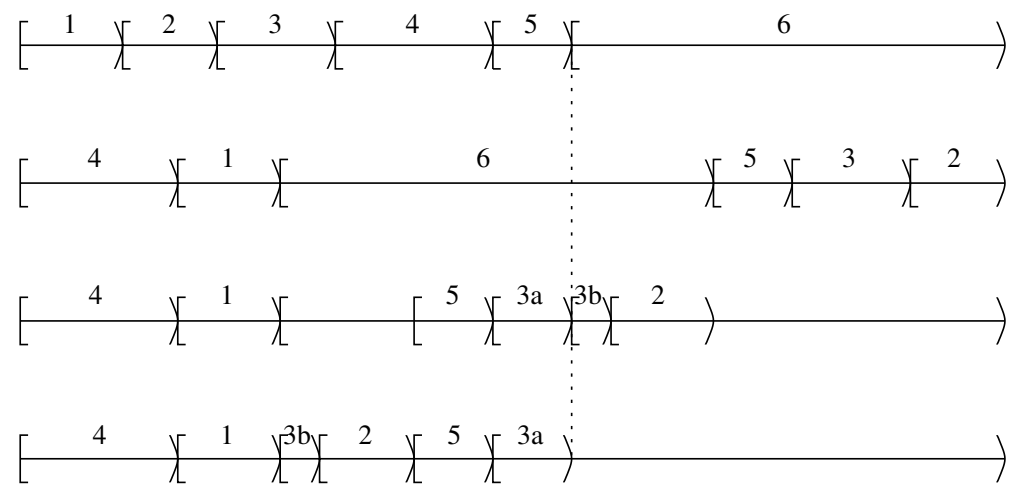

Figure 3. An example of how the intervals $\Delta_{1}, \Delta_{2}, \Delta_{3}, \Delta_{4}$, and $\Delta_{5}$ are returning to $\bigcup_{i=1}^{5} \Delta_{i}$ by the first return map.

In the first case, $\phi_{n} \Delta_{k}^{n}$ and $\Delta_{k}^{n}$ have empty intersection, while in the second case they intersect. In the former case, if $\bigcup_{i=1}^{k-1} \Delta_{i}^{n}=A_{n+1}$, then by assumption there is exactly one $i \in\{1, \ldots, k-1\}$ such that $\phi_{n} \Delta_{i}^{n} \cap A_{n+1} \neq \varnothing$ and $\phi_{n} \Delta_{i}^{n} \cap \Delta_{k}^{n} \neq \varnothing$. For all other $j \in\{1, \ldots, k-1\}$ either $\phi_{n} \Delta_{j}^{n} \subset A_{n+1}$ or $\phi_{n} \Delta_{j}^{n} \subset \Delta_{k}^{n}$. If $\phi_{n} \Delta_{j}^{n} \subset \Delta_{k}^{n}$, then clearly $\phi_{n}^{2} \Delta_{j}^{n} \subset A_{n+1}$. In case two some of the intervals are moved by $\phi_{n}$ to the right of $\phi_{n} \Delta_{k}^{n}$. Since they now lie wholly 
within $\Delta_{k}^{n}$, they will be moved as a block step by step to the left, until the left part of the block intersects $A_{n+1}$ after $m+1$ steps. The rest of the block returns after $m+2$ steps. As in the first case, there is one interval which is cut in two upon the return to $A_{n+1}$. To make it clearer, we have illustrated an example in Figure 3.

In this example, the number of intervals is 6 and $m=1$. The first part of the figure shows the original intervals. The second part shows how $\phi_{n}$ permutes these, and the third and fourth parts illustrate how the first five intervals are carried back by the first return map. The first return map acts as an interval exchange on six intervals, where the intervals are $\Delta_{1}, \Delta_{2}, \Delta_{3 a}, \Delta_{3 b}, \Delta_{4}$, and $\Delta_{5}$. So in both cases illustrated in Figure 2 one of the intervals $\Delta_{i}^{n}$ is cut upon the return to $A_{n+1}$. The rest of the intervals either return immediately or after $m+1$ or $m+2$ steps, where $m \in N \cup\{0\}$. The left part of the interval that gets cut returns after $m+1$ steps, the right part returns after $m+2$ steps. So the interval $\Delta_{i}^{n}$ is cut into $\Delta_{i}^{n+1}$ and $\Delta_{i+1}^{n+1}$. Moreover, $\Delta_{1}^{n+1}=\Delta_{1}^{n}, \ldots, \Delta_{i-1}^{n+1}=$ $\Delta_{i-1}^{n}, \Delta_{i+2}^{n+1}=\Delta_{i+1}^{n}, \ldots, \Delta_{k}^{n+1}=\Delta_{k-1}^{n}$.

This completes the proof of Theorem 3.1.

Definition 3.4. We say that an ordered Bratteli diagram which is constructed from a minimal interval exchange as described above is of interval type.

It is possible to find more requirements on the ordered Bratteli diagrams constructed above than the ones mentioned in Theorem 3.1. We will look further into this in the next chapter.

Corollary 3.5 (Putnam, [9]). The dimension group associated to a minimal interval exchange transformation is isomorphic as an abstract group to $Z^{m+1}$, where $m$ is the number of distinct orbits in $\mathscr{D}(T)$.

Proof. This follows by (ii) and (iii) of the theorem, since by (ii) the number of vertices at level $n$ becomes $m+1$ for all $n$ sufficiently large, and by (iii) the determinant of the incidence matrices becomes equal to 1. By Section 2.4 it then follows that the dimension group, as an abstract group, is isomorphic to $Z^{m+1}$.

\section{Checking that a simple ordered Bratteli diagram corresponds to an interval exchange transformation}

Assume that we are given a simple ordered Bratteli diagram satisfying the requirements of Theorem 3.1 and that we want to check whether it is of interval type. We first describe a necessary local condition. Suppose that we are given the part of the diagram for $V_{n-1}$ to $V_{n+1}$. Assuming that the diagram is of the right type, we know that the cylinder sets associated to the minimal paths 
from $V_{0}$ to $V_{n+1}$ correspond to intervals in $A_{n+1}$, and these intervals are going to be permuted in some fashion. By taking $E_{n+1}$ into account, we will get some restrictions on which permutations are allowed. Furthermore, these permutations will be lifted injectively by $E_{n+1}$ to permutations on $V_{n}$. Similarly, $E_{n}$ will give restrictions on the permutations on $V_{n-1}$. If any two consecutive levels always are consistent in the sense that a legal permutation on $V_{n-1}$ can be reached by lifting a legal permutation from $V_{n+1}$, we say that the diagram is locally consistent with being of interval type. However, the lifting process described here may stop after an arbitrary number of steps, so we need a more global approach of finding all permutations simultaneously.

We will now describe a procedure that makes it possible to determine whether a given simple ordered Bratteli diagram is of interval type. By telescoping the diagram, we may assume that there is at least one edge between each vertex of $V_{n}$ and each vertex of $V_{n+1}$ for all $n \geq 1$. If the diagram is of interval type, this means that the total length of all intervals corresponding to level $n+1$ is less than or equal to the length of the shortest of the intervals corresponding to level $n$. We use $E_{n+1}$ to determine how the intervals of $V_{n}$ are permuted. Let $V_{n}=\left\{v_{1}, \ldots, v_{m}\right\}$ and let $\left\{\Delta_{1}^{n}, \ldots, \Delta_{m}^{n}\right\}$ be the corresponding intervals. To determine how $\Delta_{i}^{n}$ is moved, we consider all paths from $V_{n+1}$ to $v_{i}$. If the successor to one of these paths goes to $v_{k}$, it means that $\phi_{n}\left(\Delta_{i}^{n}\right)$ intersects $\Delta_{k}^{n}$. If there exists a successor $p$ of a path from $V_{n+1}$ to $v_{i}$ and a successor $q$ of a path from $V_{n+1}$ to $v_{j}$, with $p$ and $q$ going to different vertices at level $n$, then we can immediately deduce the relative position of $\phi_{n}\left(\Delta_{i}^{n}\right)$ and $\phi_{n}\left(\Delta_{j}^{n}\right)$. Otherwise, if all the successors of paths from $V_{n+1}$ to $v_{i}$ and $v_{j}$ go to $v_{k}$, we know that $\phi_{n}\left(\Delta_{i}^{n}\right)$ and $\phi_{n}\left(\Delta_{j}^{n}\right)$ are lying inside $\Delta_{k}^{n}$, but we do not yet know where they are placed within $\Delta_{k}^{n}$. To determine this, we may consider the iterated successors of the paths. These will determine what interval or intervals $\phi_{n}^{m}\left(\Delta_{i}^{n}\right)$ and $\phi_{n}^{m}\left(\Delta_{j}^{k}\right)$ intersect. If $\phi_{n}^{2}\left(\Delta_{i}^{n}\right)$ and $\phi_{n}^{2}\left(\Delta_{i}^{n}\right)$ intersect different intervals, we may find the relative position of $\phi_{n}^{2}\left(\Delta_{i}^{n}\right)$ and $\phi_{n}^{2}\left(\Delta_{j}^{n}\right)$ and thereby the relative positions of $\phi_{n}\left(\Delta_{i}^{n}\right)$ and $\phi_{n}\left(\Delta_{j}^{n}\right)$ in $\Delta_{k}^{n}$. If not, we carry on in the same manner and try to find the relative position of $\phi_{n}^{m}\left(\Delta_{i}^{n}\right)$ and $\phi_{n}^{m}\left(\Delta_{j}^{n}\right)$ for some $m$. If $\phi_{n}^{l}\left(\Delta_{i}^{n}\right)$ and $\phi_{n}^{l}\left(\Delta_{j}^{n}\right)$ lie in the same interval for all $l=1,2, \ldots, m-1$, but $\phi_{n}^{m}\left(\Delta_{i}^{n}\right)$ and $\phi_{n}^{m}\left(\Delta_{j}^{n}\right)$ do not lie in the same interval, then we may determine the relative position of $\phi_{n}^{m}\left(\Delta_{i}^{n}\right)$ and $\phi_{n}^{m}\left(\Delta_{j}^{n}\right)$. Since the order of points in the same interval is preserved by the interval exchange, we may carry this order back to determine the relative positions of $\phi_{n}\left(\Delta_{i}^{n}\right)$ and $\phi_{n}\left(\Delta_{j}^{n}\right)$ in $\Delta_{k}^{n}$.

We claim that we always will be able to decide the relative position of $\phi_{n}^{m}\left(\Delta_{i}^{n}\right)$ and $\phi_{n}^{m}\left(\Delta_{j}^{n}\right)$ for some $m$. To see this, choose $l$ to be the least integer such that $\phi_{n}^{l}\left(\Delta_{i}^{n}\right)$ or $\phi_{n}^{l}\left(\Delta_{j}^{n}\right)$ contains some path which is maximal from $V_{0}$ to $V_{n+1}$. (We have identified the clopen sets $\phi_{n}^{l}\left(\Delta_{i}^{n}\right)$ and $\phi_{n}^{l}\left(\Delta_{j}^{n}\right)$ with the corresponding clopen sets in the Bratteli diagram.) Let us assume that $\phi_{n}^{l}\left(\Delta_{i}^{n}\right)$ 
contains such a path. Then, by the definition of the Bratteli-Vershik map, we must conclude that $\phi_{n}^{l+1}\left(\Delta_{i}^{n}\right)$ must intersect $\cup_{k=1}^{m} \Delta_{k}^{n+1}$. But since the total length of these intervals is less than or equal to the length of $\phi_{n}^{l}\left(\Delta_{j}^{n}\right), \phi_{n}^{l+1}\left(\Delta_{j}^{n}\right)$ cannot fit entirely within $\cup_{k=1}^{m} \Delta_{k}^{n+1} \backslash \phi_{n}^{l+1}\left(\Delta_{i}^{n}\right)$, and we must conclude that $\phi_{n}^{l+1}\left(\Delta_{i}^{n}\right)$ lies to the left of $\phi_{n}^{l+1}\left(\Delta_{j}^{n}\right)$. It follows that the interval exchange on $V_{n}$ is fully determined from $E_{n}$.

We conclude that telescoping the diagram and assuming that it is of interval type, we get unique interval permutations in $V_{1}, V_{2}, V_{3}$, etc. If these are consistent, the diagram is of interval type. If at least two of them are inconsistent, the diagram is not of interval type.

Note that while the permutation of the intervals may be deduced from the diagram, in general their lengths cannot. It is easy to see why this must be so: If $(X, \phi)$ is a non-uniquely ergodic interval exchange transformation (see [2] for examples), one may construct a new interval exchange transformation $(Y, \psi)$ by using the same permutation but rescaling the lengths of the intervals to a different invariant measure for $(X, T)$. Then $(Y, \psi)$ has the same BratteliVershik diagram as $(X, \phi)$.

\section{An application: interval exchange transformations over quadratic fields}

Let $T$ be a minimal interval exchange transformation on $n$ intervals. If we consider the induced map, $T^{\prime}$, on one of these intervals, it is also (as explained earlier) a minimal interval exchange transformation on $n$ intervals. We may continue and consider the induced transformation on one of these intervals, and so on. An interval exchange obtained in this way is called a descendant of $T$, and we call $T^{\prime}$ a first generation descendant of $T$. It is clear that the number of m-th generation descendants is $n^{m}$.

Let $\mathscr{R}$ be a quadratic number field, i.e.,

$$
\mathscr{R}=\mathrm{Q}[\sqrt{d}]=\{p+q \sqrt{d}: p, q \in \mathrm{Q}\},
$$

where $d$ is a square-free integer. We say that an interval exchange transformation is defined over a quadratic number field if the lengths of the intervals are contained in some quadratic number field $\mathscr{R}$.

Boshernitzan and Carroll [1] have recently proved that if an interval exchange transformation is defined over a quadratic number field, then its family of descendants contains only a finite number of unique interval exchange transformations up to rescaling. Combining this result with our model, we can prove the following statement.

THEOREM 5.1. A minimal interval exchange transformation over a quadratic field is conjugate to a substitution minimal system. 
PRoof. It is known that any substitution minimal system has a BratteliVershik model with a periodic Bratteli diagram and that the Cantor minimal system associated to any periodic properly ordered Bratteli diagram is conjugate to a substitution system or a stationary odometer system [3], [5]. Hence to prove the theorem, we only need to show that the diagram we get by our construction is periodic in the case that the interval exchange is over a quadratic field. (Recall that interval exchange transformations are expansive, and so are never odometers.)

We may assume, by telescoping the diagram, that level $n+1$ is constructed from the induced map on the first interval from level $n$ for all $n$. This is a consistent way of making descendants of the original interval exchange, and so as soon as the interval exchange repeats (according to the result mentioned above), we conclude that the resulting Bratteli diagram will be periodic.

We can also prove a partial converse to the above in the case when a substitution minimal system has rational rank 2 .

Definition 5.2. The rational rank of a uniquely ergodic Cantor minimal system is the dimension of the rational vector space spanned by the values of the invariant probability measure on clopen sets.

In the above definition, one might equivalently consider the range of the state corresponding to the measure, on the dimension group. For interval exchange transformations this is again equivalent to the span of the lengths of the original intervals.

Theorem 5.3. Let $(X, \phi)$ be a substitution minimal system. Assume that $(X, \phi)$ has rank 2 , so that the range of the unique invariant probability measure $\mu$ is contained within $Q+Q \alpha$, for some irrational $\alpha$ in the range of $\mu$. Then $\alpha$ is a quadratic irrational.

Proof. Since $(X, \phi)$ is a substitution minimal system, it has a periodic Bratteli-Vershik diagram $(V, E)$.

Recall the definition of the dimension group $G=K_{0}(V, E)$ as a direct limit of abelian groups of the form $Z^{\left|V_{i}\right|}$. We define an endomorphism $\rho$ on the dimension group as follows: For $g \in K_{0}(V, E)$ represent $g$ as an element of $Z^{\left|V_{n}\right|}$. Then $\rho(g)$ is represented by the same vector, but at level $n+1$.

It is clear that this is a well-defined, positive endomorphism, as the periodicity of the diagram ensures that whether the limit of a vector is 0 is independent of its position in the diagram, and similarly for positivity.

Moreover, the infinitesimal subgroup is mapped into itself by $\rho$, so that $\rho$ gives a well defined endomorphism $\rho^{\prime}$ on $G / \operatorname{Inf}(G)$. Because of unique ergodicity, $G / \operatorname{Inf}(G)$ can be identified with a subgroup of R, namely the 
range on $K_{0}(V, E)$ of the state corresponding to the invariant measure $\mu$ (and also denoted by $\mu$ ); by assumption, this is a subgroup of $Q+Q \alpha$.

Any non-zero positive endomorphism of a subgroup of $\mathrm{R}$ is given by multiplication by some positive real number. Let $\beta$ be the number corresponding to $\rho^{\prime}$. Since $1, \alpha \in \mu\left(K_{0}(V, E)\right)$, we have $\beta, \alpha \beta \in \mu\left(K_{0}(V, E)\right)$. This gives equations

$$
\begin{array}{r}
\beta=x 1+y \alpha \\
\beta \alpha=z 1+w \alpha
\end{array}
$$

with $x, y, z, w \in \mathrm{Q}$. Combining these, we get

$$
y \alpha^{2}+(x-w) \alpha-z=0
$$

We see that $\alpha$ will be quadratic unless $y=z=0$ and $x=w$, which is equivalent to $\beta$ being rational. So, if we can show that $\beta$ must be irrational, we will be done.

Assume ad absurdum that $\beta$ is rational. By the rank two assumption, let $g_{1}, g_{2} \in K_{0}(V, E)$ be such that $\mu\left(g_{1}\right) / \mu\left(g_{2}\right) \notin Q$, and let $N>1$ be a level at which both of these are represented as elements $u_{1}, u_{2}$ of $Z^{\left|V_{N}\right|}$.

Let $\mu_{N}$ be the vector representing $\mu$ at the level $N$, so that

$$
\begin{aligned}
& \mu\left(g_{1}\right)=\left\langle\mu_{N}, u_{1}\right\rangle \\
& \mu\left(g_{2}\right)=\left\langle\mu_{N}, u_{2}\right\rangle,
\end{aligned}
$$

where $\langle\bullet, \bullet\rangle$ denotes inner product of vectors. If $A$ is the incidence matrix of the periodic Bratteli-Vershik diagram (for all levels except the top one), then we have

$$
A^{T} \mu_{N}=\mu_{N-1}=\frac{\mu_{N}}{\beta} .
$$

Choose a basis $\left\{\alpha_{1}, \alpha_{2}\right\}$ for $Q+Q \alpha$ such that

$$
\mu_{N}=\mu_{N}^{1} \alpha_{1}+\mu_{N}^{2} \alpha_{2}
$$

where $\mu_{N}^{1}, \mu_{N}^{2} \in \mathrm{Q}^{\left|V_{N}\right|}$ have positive coordinates (Since each coordinate of $\mu_{N}$ is positive, this is possible - see Figure 4.)

Now we have for $i=1,2$ (since $\beta$ is rational)

$$
A^{T} \mu_{N}^{i}=\frac{\mu_{N}^{i}}{\beta} .
$$




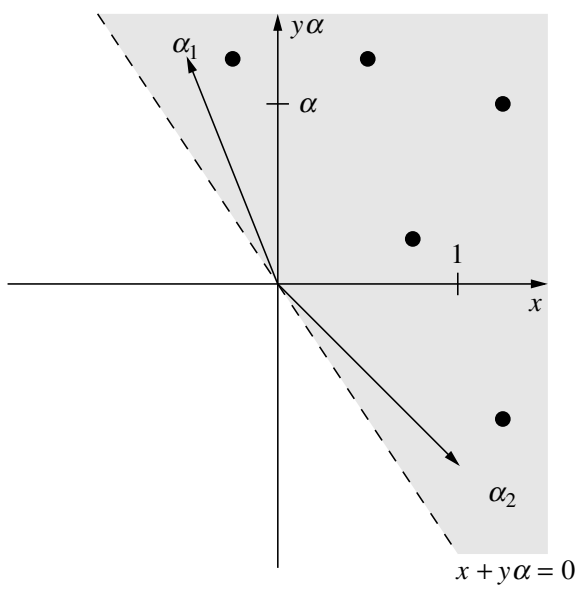

FIGURE 4. For any finite number of positive elements in $Q+Q \alpha$ one may find a basis $\left\{\alpha_{1}, \alpha_{2}\right\}$ with respect to which all of them have positive coefficients.

Therefore, $\mu_{N}^{1}, \mu_{N}^{2}$, and $\mu_{N}$ are all Perron-Frobenius eigenvectors of $A^{T}$, and thus proportional. But then the coordinates of $\mu_{N}$ must have rational ratios, which contradicts the irrationality of $\left\langle\mu_{N}, u_{1}\right\rangle /\left\langle\mu_{N}, u_{2}\right\rangle$.

\section{REFERENCES}

1. Boshernitzan, M. D. and Carroll, C. R., An extension of Lagrange's theorem to interval exchange transformations over quadratic fields, J. Analyse Math. 72 (1997), 21-44.

2. Cornfeld, I. P., Fomin, S. V., and Sinaǐ, Ya. G., Ergodic Theory, Grundlehren Math. Wiss. 245, 1982.

3. Durand, F., Host, B., and Skau, C., Substitution dynamical systems, Bratteli diagrams, and dimension groups, Ergodic Theory Dynam. Systems 19 (1999), no. 4, 953-993.

4. Effros, E. G., Dimensions and $C^{*}$-algebras, CBMS Reg. Conf. Ser. Math. 46, 1981.

5. Forrest, A. H., K-groups associated with substitution minimal systems, Israel J. Math. 98 (1997), 101-139.

6. Giordano, T., Putnam, I. F., and Skau, C. F., Topological orbit equivalence and $C^{*}$-crossed products, J. Reine Angew. Math. 469 (1995), 51-111.

7. Herman, R. H., Putnam, I. F., and Skau, C. F., Ordered Bratteli diagrams, dimension groups, and topological dynamics, Internat. J. Math. 3 (1992), 827-864.

8. Keane, M., Interval exchange transformations, Math. Z. 141 (1975), 25-31.

9. Putnam, I. F., The $C^{*}$-algebras associated with minimal homeomorphisms of the Cantor set, Pacific J. Math. 136 (1989), 329-353. 